\title{
Inhibition of autophagy enhances SMI-4a-induced growth inhibition and apoptosis of melanoma cells
}

\author{
Lei Chen ${ }^{1}$, Da-lun Lv ${ }^{1 *}$, Wen-bei Liu ${ }^{2}$, Wei Ding ${ }^{1}$, Wei Zhang ${ }^{1}$, He-li wang ${ }^{1}$, Shuai \\ Wang $^{1}$ \\ ${ }^{1}$ Department of Burns and Plastic Surgery, ${ }^{2}$ Dermatological Department, First Affiliated Hospital of Wannan Medical College, \\ Jinghu District, Wuhu City, Anhui Province, 241000, China
}

*For correspondence: Email: Lvdalun89@163.com

\begin{abstract}
Purpose: To investigate the exact role of the proviral integration site for Moloney murine leukemia virus-1 (PIM-1) on autophagy as well as the underlying molecular mechanisms in melanoma.

Methods: mRNA expression levels in A375 and G361 human melanoma cell lines were measured using reverse transcription-quantitative polymerase chain reaction (RT-qPCR). Enzyme-linked immunosorbent (ELISA) and western blotting assays were applied to determine protein expression levels, while cell viability was evaluated using Cell Counting Kit 8 and colony formation assay. Flow cytometric analysis and caspase $3 / 7$ activity assay were used to assess apoptosis.

Results: The results show that pharmacological inhibition of PIM-1 with its potent inhibitor (SMI-4a) suppressed cell viability and induced apoptosis in melanoma cell lines A375 and G361. SMI-4a also induced autophagy through inhibition of the phosphoinositide 3-kinase (PI3K)/AKT/mammalian target of rapamycin (mTOR) axis in melanoma cells. Furthermore, chloroquine, an inhibitor of autophagy, potentiated the SMI-4a-induced inhibition of tumour growth and promotion of apoptosis in melanoma cells in vitro and in vivo.

Conclusions: These results suggest that SMI-4a induces protective autophagy via PI3KJAKT/mTOR signaling pathway in melanoma cells. Thus, a combination of SMI-4a and an inhibitor of autophagy might be a novel approach to melanoma therapy.
\end{abstract}

Keywords: Apoptosis, Autophagy, Cell viability, Melanoma, PIM-1, SMI-4a

\begin{abstract}
This is an Open Access article that uses a funding model which does not charge readers or their institutions for access and distributed under the terms of the Creative Commons Attribution License (http://creativecommons.org/licenses/by/4.0) and the Budapest Open Access Initiative (http://www.budapestopenaccessinitiative.org/read), which permit unrestricted use, distribution, and reproduction in any medium, provided the original work is properly credited.
\end{abstract}

Tropical Journal of Pharmaceutical Research is indexed by Science Citation Index (SciSearch), Scopus, International Pharmaceutical Abstract, Chemical Abstracts, Embase, Index Copernicus, EBSCO, African Index Medicus, JournalSeek, Journal Citation Reports/Science Edition, Directory of Open Access Journals (DOAJ), African Journal Online, Bioline International, Open-J-Gate and Pharmacy Abstracts

\section{INTRODUCTION}

Melanoma, arising from epidermal melanocytes, is a highly aggressive skin cancer type with mounting substantial incidence in the past 30 years [1]. It is associated with early metastasis, poor prognosis, very low 5-year survival rate, and high mortality. Therefore, it is imperative to explore the mechanism of melanoma pathogenesis, identify novel therapeutic targets, and develop effective strategies for melanoma treatment.

PIM-1, which belongs to the PIM family, is 
overexpressed in numerous solid tumours [2]. A growing body of evidence has demonstrated that atopic expression of PIM-1 in cancer cells is responsible for tumour growth and metastasis [3]. Inhibition of PIM-1 by knockdown studies can reduce proliferation and viability in preclinical models of melanoma [4]. However, the exact role of PIM-1 as a therapeutic target in melanoma is not clearly understood.

Autophagy is widely implicated in various biological activities such as programmed cell death, differentiation, innate and adaptive immunity, aging, tumourigenesis, and tumour progression [5]. Autophagy plays dual and contradictory roles in cancer. Recent findings showed that pharmacotherapy induced cell autophagy, which in turn promoted the survival of tumour cells [6]. Inhibition of autophagy potentiated arginase-induced cytotoxicity in breast cancer cells [7]. Therefore, it is critical and necessary to investigate the functions of autophagy in certain contexts.

To better understand the biological function of PIM-1 in melanoma and to determine the therapeutic application of PIM-1 inhibitors in melanoma, we investigated whether pharmacological inhibition of PIM-1 could induce autophagy in melanoma, and explored the underlying mechanisms.

\section{EXPERIMENTAL}

\section{Cell culture}

Human melanoma cell lines A375 and G361 were purchased from the Cell Bank of the Chinese Academy of Sciences (Shanghai, China). The cells were cultured in Dulbecco's Modified Eagle Medium supplemented with $10 \%$ FBS, 100 units $/ \mathrm{mL}$ penicillin, and $100 \mu \mathrm{g} / \mathrm{mL}$ streptomycin (Gibco BRL, Grand Island, NY). The cells were grown at $37{ }^{\circ} \mathrm{C}$ in a humidified atmosphere with $5 \% \mathrm{CO}_{2}$.

\section{Cell viability assay}

Cell survival was evaluated with Cell Counting Kit 8 (CCK8, Beyotime, Shanghai, China), according to the manufacturer's instructions, and the colony formation assay. For the CCK8 assay, cells $(3 \times$ $10^{3}$ cells/ well) were seeded into 96-well plates and then treated with SMI-4a, with double dilution from $10 \mu \mathrm{M}$ (Sigma-Aldrich, Merck $\mathrm{KGaA}$, Darmstadt, Germany). The viability of the cells was analyzed after incubation for 24,48 , and $72 \mathrm{~h}$, respectively. The optical density values were read at $450 \mathrm{~nm}$ using a microplate reader (Thermo, USA). For the colony formation assay,
600 cells were seeded into six-well plates and treated with SMI-4a $(0.3$ and $1 \mu \mathrm{M})$ at $37^{\circ} \mathrm{C}$ for 10 days, followed by fixation and staining with $0.5 \%$ crystal violet and methanol. The mean value of three independent experiments were calculated.

\section{Flow cytometry}

For the detection of apoptosis, A375 and G361 cells were treated with SMI-4a $\left(\begin{array}{lll}1 \mu M & \text { in }\end{array}\right.$ complete medium for $48 \mathrm{~h}$. Cells were collected, resuspended in binding buffer, and stained with annexin-V-FITC/PI apoptosis detection kit (KGI Biotech, Nanjing, China) according to the manufacturer's protocol The stained cells were resuspended for flow cytometry analysis. (FACSCalibur, BD Biosciences, San Jose, California, USA). For the detection of autophagic body production, $\mathrm{A} 375$ and $\mathrm{G} 361$ cells were treated with SMI-4a $(3,1$, and $0.3 \mu \mathrm{M})$ for $48 \mathrm{~h}$. Cells were collected and stained with acridine orange $(1 \mu \mathrm{g} / \mathrm{mL})$ and protected from light for 15 $\mathrm{min}$ at room temperature. The stained cells were resuspended for flow cytometry analysis.

\section{Caspase $3 / 7$ activity assay}

Cells were plated into 96-well plates and treated with SMI-4a $(1 \mu \mathrm{M})$ and/or CQ $(3 \mu \mathrm{M})$ for $48 \mathrm{~h}$. $\mathrm{CQ}$ is a lysosome inhibitor which blocks the fusion of autophagosomes and lysosomes [8]. Caspase-Glo® 3/7 Reagent was added to the wells, and luminescence was recorded using the Caspase-Glo ${ }^{\circledR} \quad 3 / 7$ Assay kit (Promega Corporation, Madison, WI, USA). The data were calculated at a wavelength of $499 \mathrm{~nm}$ with a microplate reader. Values from wells containing culture medium alone served as background.

\section{Protein isolation and western blot analysis}

Total protein was extracted from cells using RIPA Buffer (Cell Signaling Technology, Danvers, MA, USA). The protein concentration was assessed with a bicinchoninic acid (BCA) assay kit from EMD Millipore (Billerica, MA, USA). Protein was separated with SDS polyacrylamide gel electrophoresis, and electrophoretically transferred onto PVDF membranes. The primary antibodies used were as following: LC3B antibody (Cell Signaling Technology, 1:1000, no. 3868), Beclin-1 antibody (Cell Signaling Technology, 1:1000, no. 3495), Atg5 antibody (Cell Signaling Technology, 1:1000, no. 9980), AKT antibody (Cell Signaling Technology, 1:1000, no. 4685), p-AKT antibody (Cell Signaling Technology, 1:1000, no. 4060), mTOR antibody (Cell Signaling Technology, 1:1000, no. 
2983), p-mTOR antibody (Cell Signaling Technology, 1:1000, no. 5536), ) and GAPDH antibody (Beyotime, 1:5000, no. AG019). Bands were developed using the RapidStep ${ }^{\mathrm{TM}} \mathrm{ECL}$ Reagent (EMD Millipore) according to the manufacturer's directions.

\section{Tumour xenografts}

A375 cells $\left(3 \times 10^{6}\right)$ were subcutaneously inoculated into the right flank of five six-week old nude mice (Charles River Laboratories). Measurement of tumor length and width was performed every three days with calipers, and tumour volume was calculated using the formula: volume $=1 / 2 \times$ length $\times$ width. The study was approved by Animal Ethic Committee of Scientific research IRB of Wannan Medical College Yijishan Hospital (approval ref no. 2016-13). All experimental procedures involving animals were performed according to the Principles of Laboratory Animal Care (NIH publication no. 8523, revised 1985) and/or the Declaration of Helsinki promulgated in 1964 as amended in $1996[9,10]$.

\section{Statistical analysis}

All the data are presented as mean \pm standard deviation (SD, $n=3)$. Statistical analysis was performed with SPSS 13.0 software (SPSS, Inc., Chicago, IL, USA). The Student $t$ test or ANOVA were used to analyze differences between the different treatment groups. $P$ value below 0.05 was considered statistically significant.

\section{RESULTS}

PIM-1 specific inhibitor, SMI-4a, suppressed cell viability in melanoma cells

To confirm whether the pharmacological inhibition of PIM-1 with SMI-4a had a similar effect on cell survival, human melanoma cells A375 and G361 were treated with SMI-4a. CCK8 cytotoxicity assays indicated that SMI-4a showed significant inhibitory effect on cell viability in a time- and dose-dependent manner in A375 and G361 melanoma cells (Figure 1A). The antiproliferative effect of SMI-4a was also examined using the colony formation assay. Consistent with the cell viability results, treatment with SMI4a remarkably inhibited cell colony formation ability in a dose-dependent fashion in A375 and G361 cells $(p<0.001$, Figure 1B). These results demonstrate that SMI-4a inhibits cell viability in A375 and G361 melanoma cells in vitro.
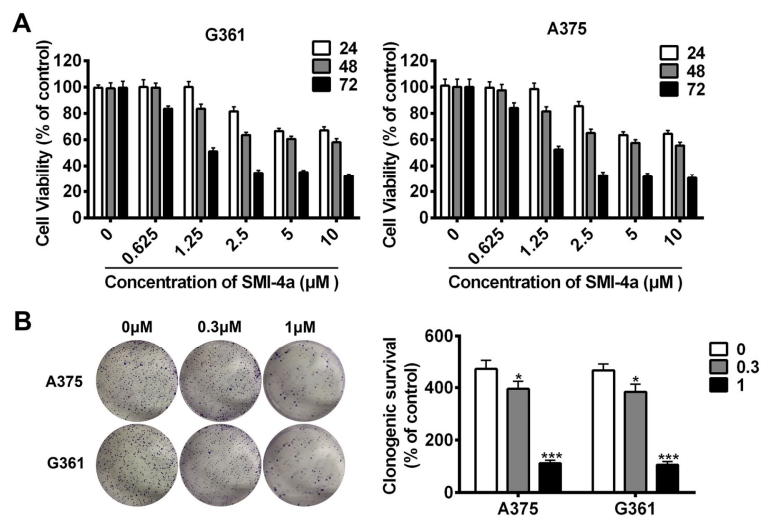

Figure 1: PIM-1 specific inhibitor, SMI-4a, suppresses melanoma cell viability. (A) SMl-4a suppress melanoma cell viability. A375 cells and G361 cells were incubated with a concentration gradient of SMI$4 \mathrm{a}$ for 24,48 , and $72 \mathrm{~h}$. For a negative control, $0.1 \%$ DMSO was used. (B) SMI-4a reduced clonogenic survival in melanoma cells. Cells were treated with SMI-4a ( 1 and $0.3 \mu \mathrm{M})$ for 10 days. Treatment with $0.1 \%$ DMSO served as the control. Colonies were fixed, stained with crystal violet and quantified; " $p<$ 0.05 and ${ }^{* * *} p<0.001$, when compared with the negative control. Data are expressed as mean \pm SD

\section{Inhibition of PIM-1 with SMI-4a promotes apoptosis in melanoma cells}

Next, we further investigated whether SMI-4a exerted an effect on cell apoptosis. We found that SMI-4a-treated A375 cells displayed greater numbers of early apoptotic cells as well as late apoptotic/secondary necrotic cells relative to the control group (23.4 vs. 4.46; $p<0.001$; Figure 2 A). A similar effect of SMI-4a on apoptosis was observed in $\mathrm{G} 361$ cells (22.0 vs. 2.91; $p<0.001$ Figure 2A). An increase in apoptosis was further evidenced by the detection of upregulated caspase $3 / 7$ activity, relative to the control group, in A375 cells ( 157 vs. 46; $p<0.001$; Figure 2 B) and G361 cells (188 vs. 32; $p<0.001$; Figure 2 $B)$. These results demonstrate that SMI-4a induced apoptosis in A375 and G361 melanoma cells in vitro.

\section{SMI-4a induced autophagy via PI3K/AKT/ mTOR signaling pathway}

To investigate the effects of SMI-4a on autophagy, autophagic body production was assessed with acridine orange staining. It was found that SMI-4a treatment significantly increased autophagic body production in a dosedependent manner relative to that of the control group, in A375 cells and G361 cells $(p<0.01$, Figure 3 A). Moreover, several critical determinants of autophagy include Atg5, LC3, and Beclin-1 were examined. Our results showed that SMI-4a resulted in an increase in the protein levels of LC3-II, Atg5, and Beclin1 in a dose- 
dependent manner in A375 and G361 (Figure 3 B). SMI-4a induces the autophagy of melanoma cells. To further investigate the molecular mechanisms underlying SMI-4a triggered autophagy, changes in PI3K/AKT/mTOR signaling upon treatment of $\mathrm{SMI}-4 \mathrm{a}$ were assessed using western blot analysis. Interestingly, SMI-4a treatment caused a reduction in the phosphorylation levels of Akt and mTOR, but showed no effect on total ATK and mTOR in A375 and G361 cells (Figure 3 C).
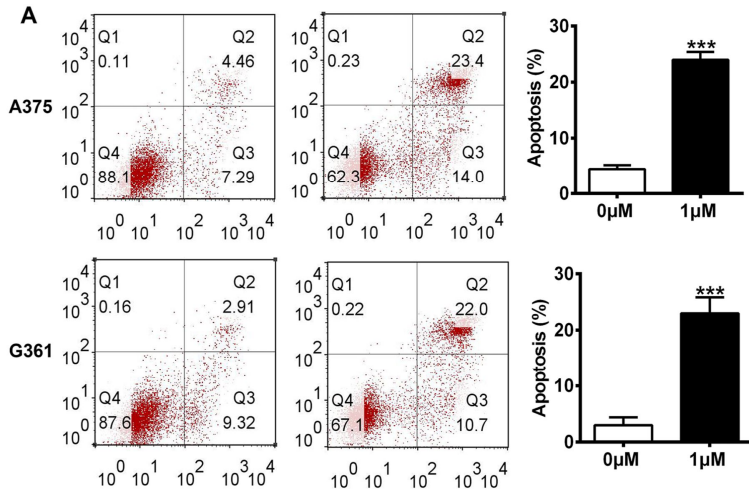

B
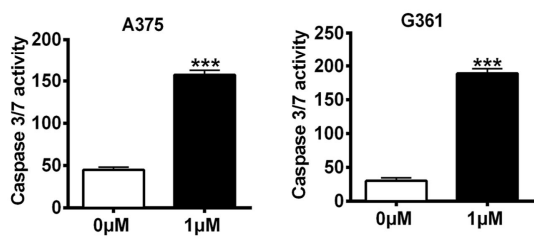

Figure 2: The PIM-1-specific inhibitor SMI-4a promotes apoptosis. (A) SMI-4a promoted the early proportion of apoptotic and late apoptotic/secondary necrotic phenotype in A375 cells and G361 cells. Cells were treated with $1 \mu \mathrm{M} \mathrm{SMI}-4 \mathrm{a}$ for $48 \mathrm{~h}$, and apoptosis was measured by PI-FACS analysis. DMEM $10 \%$ FBS with $0.1 \%$ DMSO was used as negative control. (D) SMI-4a induced caspase 3/7 activity. Cells were treated with $1 \mu \mathrm{M} \mathrm{SMI}-4 \mathrm{a}$ for $48 \mathrm{~h}$, and caspase $3 / 7$ activity was measured; ${ }^{* * *} p<0.001$, when compared with negative control. Data are expressed as the mean $\pm \mathrm{SD}$

\section{Blocking autophagy enhanced the anti- tumour effect of SMI-4a in vitro}

To verify the effect of autophagy on the SMI-4ainduced inhibition of cell viability and increase of apoptosis, an inhibitor of autophagy, chloroquine (CQ), was used to suppress autophagy in human melanoma cells. As shown in Figure 4A, the combination of SMI-4a with $\mathrm{CQ}$ exerted a greater inhibitory effect on cell survival, relative to that of each individual agent ( $p<0.001$, Figure 4 A). Consistent with this, western blotting showed that $\mathrm{CQ}$ enhanced the SMI-4a-induced expression levels of LC3B-II and Beclin-1, indicating that $C Q$ inhibited autophagy in SMI-4a-treated cells (Figure 4 B). Furthermore, inhibition of autophagy with $\mathrm{CQ}$ also enhanced SMI-4ainduced caspase $3 / 7$ activity in melanoma cells ( $p$ $<0.001$, Figure $4 \mathrm{C}$ ).

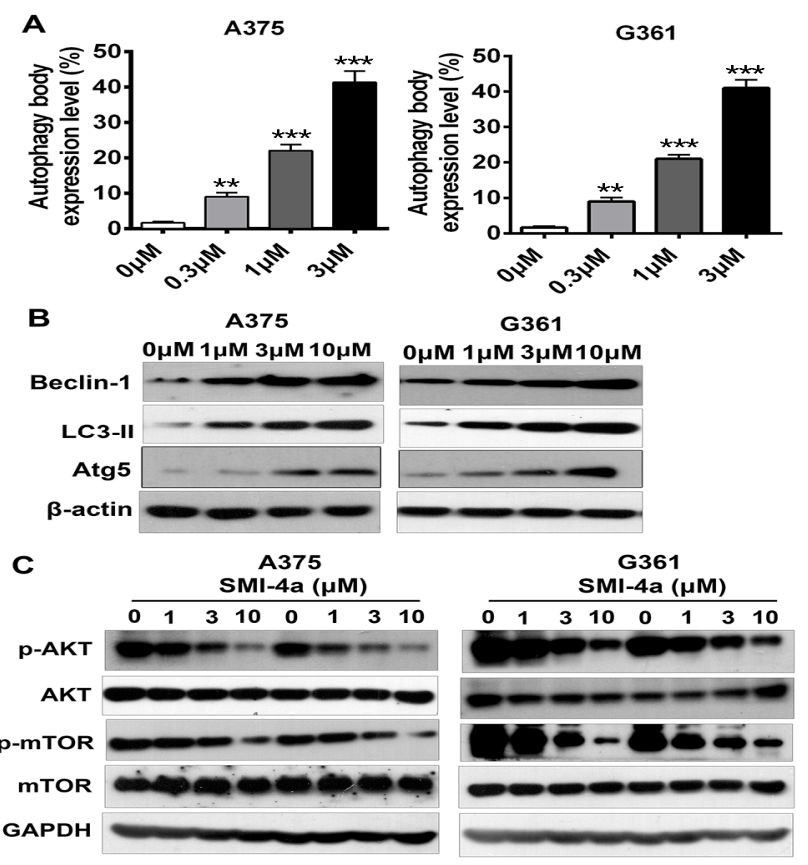

Figure 3: The PIM-1-specific inhibitor SMI-4a induces autophagy. (A) SMl-4a-induced autophagic body production. A375 and G361 cells were treated with SMI-4a $(3,1$, and $0.3 \mu \mathrm{M})$ for $48 \mathrm{~h}$. The level of autophagic body production was measured using flow cytometry analysis. (B) A375 and G361 cells were incubated with different concentrations of SMI-4a (3, 1 and $0.3 \mu \mathrm{M})$ for $24 \mathrm{~h}$. The protein expression levels of LC3B, Atg5, and Beclin1 were evaluated with western blot. (C) A375 and G361 cells were treated with different concentrations of SMI-4a at $(10,3$, and $1 \mu \mathrm{M})$ for $24 \mathrm{~h}$. The protein expression of P-AKT, total AKT, P-mTOR. and total mTOR were measured by western blot. ${ }^{* *} p<0.01$ and ${ }^{* * *} p<0.001$, when compared with the negative control. Data are expressed as mean \pm SD

Inhibition of autophagy potentiated SMI-4ainduced growth inhibition of melanoma cells in vivo

Given that SMI-4a and chloroquine showed a combinatorial effect on inhibition of cell survival and induction of apoptosis in vitro, we investigated whether SMI-4a and chloroquine showed this combinatorial effect in vivo. As expected, SMI-4a treatment significantly inhibited tumour growth compared to treatment with vehicle alone $(p<0.001)$, whereas chloroquine was not active. The combination of SMI-4a and chloroquine was more efficacious than each individual agent $(p<0.01$, Figure 5$)$. 


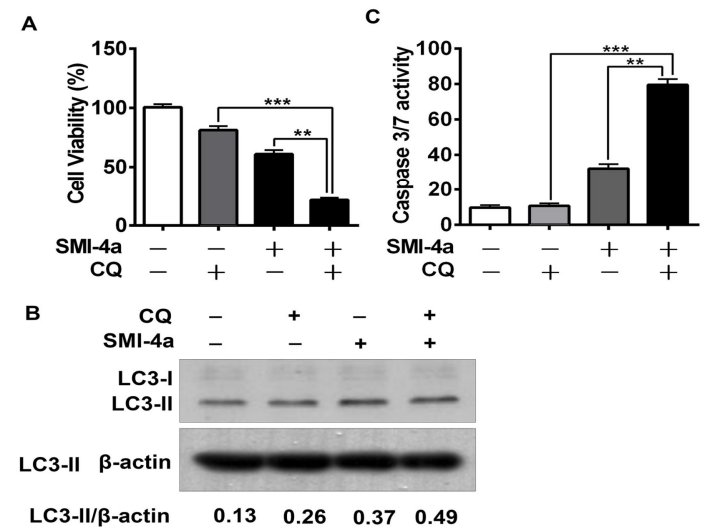

Figure 4: Inhibition of autophagy potentiated SMI-4ainduced growth inhibition and apoptosis. (A) Cell viability was evaluated with the CCK8 assay in melanoma cells that were treated with $1 \mu \mathrm{M} \mathrm{SMI}-4 a$ in the absence or presence of $3 \mu \mathrm{M}$ chloroquine for $48 \mathrm{~h}$. (B) Western blotting was used to analyse the protein levels of LC3B and Beclin-1. (C) Caspase 3/7 activity was analysed in melanoma cells treated with $1 \mu \mathrm{M}$ $\mathrm{SMI}-4 \mathrm{a}$ in the absence or presence of $3 \mu \mathrm{M}$ chloroquine for $48 \mathrm{~h} ;{ }^{* *} p<0.01$ and ${ }^{* * *} p<0.001$, when compared with the negative control. Data are expressed as mean \pm SD

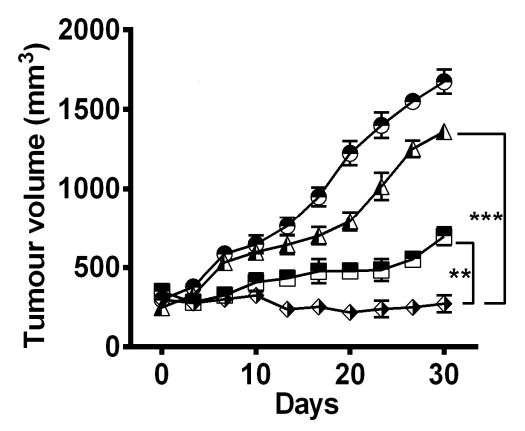

Figure 5: Inhibition of autophagy enhanced the antitumour effect of SMl-4a in a melanoma xenograft model. Melanoma A375 cells were implanted in the right flank to induce tumour growth, as described in the Methods. When the tumour volume was 150-200 $\mathrm{mm}^{3}$, the mice were randomly grouped into four groups of 6 mice each. Mice in the four groups were given orally administrations of $0.5 \% \mathrm{MC}$ (vehicle control), SMl-4a (15 mg/kg, po, qid), chloroquine (30 $\mathrm{mg} / \mathrm{kg}$, po, qid), or SMl-4a plus chloroquine, respectively. The tumour volume in each mouse was measured using calipers every 3 days, and average tumour volume for each group was calculated. vehicle, $\square$ : SMI, $\neg$ : CQ, $₫$ : SMI+CQ.

\section{DISCUSSION}

Melanoma is an aggressive type of skin cancer. It is common in adults and has multiple underlying pathogenic mechanisms. Despite progress in the treatment of melanoma, the prognosis of patients has remained dismal [11]. Therefore, it is critical to identify the underlying molecular mechanisms in order to support the development of effective and novel treatments. PIM1 is overexpressed in several tumour types, including solid tumours and liquid malignancies, and contributes to tumour growth and metastasis [12]. Expression of PIM1 is associated with tumour aggressiveness, and it is a marker of poor prognosis in various tumours [13]. Drugs targeting PIM kinases are under clinical development to determine their potential in patients with hematologic malignancies or solid tumours. Therefore, PIM-1 kinase may be a potential target for cancer treatment.

According to data from the Cancer Genome Atlas (TCGA), it has been shown that alterations in the PIM-1 gene, including mutations, amplifications, and deletions, account for up to $8 \%$ of melanomas. An inhibitory effect of PIM-1 silencing on the proliferation of melanoma cells has been previously suggested [3]. SMI-4a is a small molecular highly selective PIM-1 inhibitor that has been found to block PIM-1 kinase activity in vitro and in vivo [14]. In this study, we demonstrated that blockade of PIM-1 with SMI4a results in a remarkable decrease in cell viability, and data from the colony formation assay was consistent with this observation. Evidence from the annexin V-PI assay and caspase 3/7 activity analysis in melanoma cells. An evidence indicated that SMI-4a could promote apoptosis in vitro. The complex interplay between PI3K/AKT/mTOR axis and the autophagic process is well documented [15-17]. The PI3K/Akt/mTOR signaling pathway negatively regulates autophagy through phosphorylation of Akt, downstream effector mTOR, and its substrate p70S6K [18]. It was further revealed that the PI3K/AKT/mTOR signaling pathway was involved in SMI-4a triggered autophagy in melanoma cells.

Increasing evidence has indicated that treatment can induce autophagy in various tumour types. However, the contradictory roles of autophagy in cancer can be a double-edged sword, and are often puzzling. Some drugs induce autophagic cell death, whereas autophagy induced by other drugs appears to function as a protective cell survival mechanism $[19,20]$. It has been reported that autophagy was responsible for drug resistance in certain contexts [21]. For instance, chemotherapeutic agents induced autophagy as a possible survival mechanism [22]. This study reports for the first time that blockade of PIM-1 with SMI-4a significantly increased autophagic body production and increased several critical determinants of autophagy, including Atg5, LC3, and Beclin-1, in a dose-dependent manner. To further to examine the contributions of autophagy 
induced by SMI-4a treatment, the autophagy inhibitor $\mathrm{CQ}$ was used to pharmacologically inhibit SMI-4a-induced autophagy. CQ functions to block lysosomal acidification and autophagosome degradation [23]. Our findings indicate that inhibition of autophagy with $\mathrm{CQ}$ could remarkably potentiate SMI-4a-induced antitumour activity in vitro and in vivo, indicating the cytoprotective role of autophagy in SMI-4a therapy for melanoma.

\section{CONCLUSION}

The findings of this study indicate that inhibition of PIM-1 with SMI-4a treatment suppresses cell survival, promote apoptosis in melanoma cells, and induce autophagy. Importantly, inhibition of autophagy enhances the potency of SMI-4a in vitro and in vivo, suggesting a cytoprotective role for SMI-4a-induced autophagy. Moreover, PI3K research indicates that SMI-4a in combination with an autophagy inhibitor might be a novel alternative for the treatment of melanoma.

\section{DECLARATIONS}

\section{Conflict of Interest}

The authors declare that there is no conflict of interest associated with this work.

\section{Contribution of authors}

We declare that this work was done by the authors named in this article and all liabilities pertaining to claims relating to the content of this article will be borne by the authors. Da-lun LV designed all the experiments and revised the paper. Lei Chen, Wen-bei Liu, Wei Ding and Wei Zhang performed the experiments, Lei Chen, Heli wang and Shuai Wang wrote the paper.

\section{REFERENCES}

1. Wu X, Yan J, Dai J, Ma M, Tang H, Yu J, Xu T, Yu H, Si L, Chi Z, et al. Mutations in BRAF codons 594 and 596 predict good prognosis in melanoma. Oncol Lett 2017; 14: 3601-3605.

2. Rang Z, Yang G, Wang YW, Cui F. miR-542-3p suppresses invasion and metastasis by targeting the proto-oncogene serine/threonine protein kinase, PIM1, in melanoma. Biochem Biophys Res Commun 2016; 474: 315-320.

3. Warfel NA, Kraft AS. PIM kinase (and Akt) biology and signaling in tumors. Pharmacol Ther 2015; 151: 41-49.

4. Shannan $B$, Watters $A$, Chen $Q$, Mollin $S$, Dörr $M$, Meggers E, XU X, Gimotty PA, Perego M, Li L. PIM kinases as therapeutic targets against advanced melanoma. Oncotarget 2016; 7: 54897-54912.

5. Levine B, and Kroemer G. Autophagy in the pathogenesis of disease. Cell 2008; 132, 27-42.

6. Yang $S$, Wang $X$, Contino $G$, Liesa $M$, Sahin E, Ying $H$, Bause A, Li Y, Stommel JM, Dell'antonio G. Pancreatic cancers require autophagy for tumor growth. Genes Dev 2011; 25: 717-729.

7. Wang Z, Shi X, Li Y, Fan J, Zeng X, Xian Z, Wang Z, Sun $Y$, Wang S, Song P. Blocking autophagy enhanced cytotoxicity induced by recombinant human arginase in triple-negative breast cancer cells. Cell Death Dis 2014; 5: e1563.

8. Levy JMM, Towers CG, Thorburn A. Targeting autophagy in cancer. Nat Rev Cancer 2017; 17: 528-542.

9. Jin K, He K, Han N, Li G, Wang H, Xu Z, Jiang H, Zhang $J$, Teng L. Establishment of a PDTT xenograft model of gastric carcinoma and its application in personalized therapeutic regimen selection. Hepatogastroenterology. 2011; 58:1814-22.

10. Lan $H$, Zheng L, Jin K, Teng L. Antitumor effect of FP3 in a breast cancer xenograft model. Exp Ther Med. 2013; 5(1):85-88.

11. Brault L, Gasser C, Bracher F, Huber K, Knapp S, Schwaller J. PIM serine/threonine kinases in the pathogenesis and therapy of hematologic malignancies and solid cancers. Haematologica 2010; 95: 1004-1015

12. Santio NM, Eerola SK, Paatero I, Yli-Kauhaluoma J, Anizon F, Moreau P, Tuomela J, Härkönen P, Koskinen PJ. Pim Kinases Promote Migration and Metastatic Growth of Prostate Cancer Xenografts. PLoS One 2015; 10: e0130340.

13. Santio NM, Vahakoski RL, Rainio EM, Sandholm JA, Virtanen SS, Prudhomme M, Anizon F, Moreau $P$, Koskinen PJ. Pim-selective inhibitor DHPCC-9 reveals Pim kinases as potent stimulators of cancer cell migration and invasion. Mol Cancer 2010; 9: 279.

14. Lin YW, Beharry ZM, Hill EG, Song JH, Wang W, Xia Z, Zhang Z, Aplan PD, Aster JC, Smith CD, Kraft AS. A small molecule inhibitor of Pim protein kinases blocks the growth of precursor T-cell lymphoblastic leukemia/lymphoma. Blood 2012; 115: 824-833.

15. Heras-Sandoval D, Pérez-Rojas JM, Hernández-Damián $J$, Pedraza-Chaverri J. The role of PI3KJAKT/mTOR pathway in the modulation of autophagy and the clearance of protein aggregates in neurodegeneration. Cell Signal 2014; 26: 2694-2701.

16. Mabuchi S, Kuroda H, Takahashi R, and Sasano T. The PI3K/AKT/mTOR pathway as a therapeutic target in ovarian cancer. Gynecol Oncol 2015; 137: 173-179.

17. Roy B, Pattanaik AK, Das J, Bhutia SK, Behera B, Singh $P$, Maiti TK. Role of PIJK/Akt/mTOR and MEK/ERK pathway in Concanavalin A induced autophagy in HeLa cells. Chem Biol Interact 2014; 210: 96-102.

18. Kim, YC, and Guan KL. mTOR: a pharmacologic target for autophagy regulation. J Clin Invest 2015; 125: 25-32. 
19. Liu L, Liao JZ, He XX, and Li PY. The role of autophagy in hepatocellular carcinoma: friend or foe. Oncotarget 2017; 8: 57707-57722.

20. Mathiassen SG, De ZioD and Cecconi F. Autophagy and the Cell Cycle: A Complex Landscape. Front Oncol 2017; 7: 51

21. Gao L, Jauregui CE, and Teng Y. Targeting autophagy as a strategy for drug discovery and therapeutic modulation. Future Med Chem 2017; 9: 335-345.
22. Kaushal GP, Kaushal V, Herzog C, and Yang C. Autophagy delays apoptosis in renal tubular epithelial cells in cisplatin cytotoxicity. Autophagy 2008; 4: 710712.

23. Su Z, Li G, Liu C, Ren S, Deng T, Zhang S, Tian Y, Liu Y, Qiu Y. Autophagy inhibition impairs the epithelialmesenchymal transition and enhances cisplatin sensitivity in nasopharyngeal carcinoma. Oncol Lett 2017; 13: 4147-4154. 